\title{
Measurement of shock wave unsteadiness using a high-speed schlieren system and digital image processing
}

\author{
D. Estruch, N. J. Lawson, D. G. MacManus, K. P. Garry, and J. L. Stollery \\ Department of Aerospace Sciences, Cranfield University, Cranfield, Bedfordshire MK43 OAL, United Kingdom
}

(Received 22 October 2008; accepted 30 November 2008; published online 29 December 2008)

\begin{abstract}
A new method to measure shock wave unsteadiness is presented. Time-resolved visualizations of the flow field under investigation are obtained using a high-speed schlieren optical system and the motion of the shock wave is determined by means of digital image processing. Information on the shock's unsteadiness is subsequently derived with Fourier analysis. A sample study on shock unsteadiness in a shock-wave/turbulent boundary-layer interaction with separation is included. The method presented enables a measure of shock unsteadiness at locations in the imaged flow field not accessible by intrusive methods. (C) 2008 American Institute of Physics. [DOI: 10.1063/1.3053361]
\end{abstract}

Shock waves are generally present in supersonic flows and constitute steep changes in the local flow properties. Downstream of a shock, static pressure, density, and temperature are increased. In some cases, such as in regions of strong viscous-inviscid interaction, shock waves can be unsteady and result in fluctuating loads that need to be predicted in many aerospace applications. ${ }^{1}$ Most previous studies on shock unsteadiness have relied on high-speed wall pressure measurements to estimate the location where the shock is in contact with a surface. ${ }^{2,3}$ This limitation may be overcome by optical techniques, but their application to high-speed flows can be restricted by the high sampling frequencies required to measure the shock positions. Previous optical-based methods have used a schlieren technique with a laser diode light source and a spatial filter to obtain timedependent voltage signals sensitive to the density gradients and measured through an array of 16 photodiodes at acquisition speeds of up to $40 \mathrm{kHz}{ }^{4}$

This paper presents a modified schlieren method incorporating the latest light-emitting diode (LED) technology and digital imaging systems, which determine the location of the shock position at frequencies in excess of the shock transients. The associated image processing methods are also presented, which allow accurate location of the shock position and analysis of the transient properties of the shock.

The schlieren technique is an optical diagnostic commonly used in supersonic and hypersonic wind tunnel testing. It is based on the principle of refraction, which depends on the change in local fluid density. ${ }^{5}$ Schlieren visualizations discriminate aerodynamic features present in the flow such as shock waves and boundary layers as brighter and darker regions in the image. ${ }^{6}$ High-speed schlieren is an advanced variance of this technique, which consists in obtaining timeresolved visualizations that provide information on the unsteady flow features. In applications to high-speed flows, this generally requires the use of a capturing device at high frame rates with short exposure times. Thus high intensity and incoherent light sources are required. ${ }^{7,8}$ The main limitation of high-speed schlieren systems to date has been obtaining a suitable light source, which must be synchronized with the capturing device. ${ }^{9}$
The present arrangement (Fig. 1) has made use of the latest technology high-power LED light source (3 W CREE Q5) in a continuous mode and in combination with direct imaging onto a high-speed digital camera (Photron APX). By using the light source in a continuous mode, a relatively simple system has been obtained. The system has been developed in the Cranfield University $2.5^{\prime \prime} \times 2.5^{\prime \prime}$ Super-

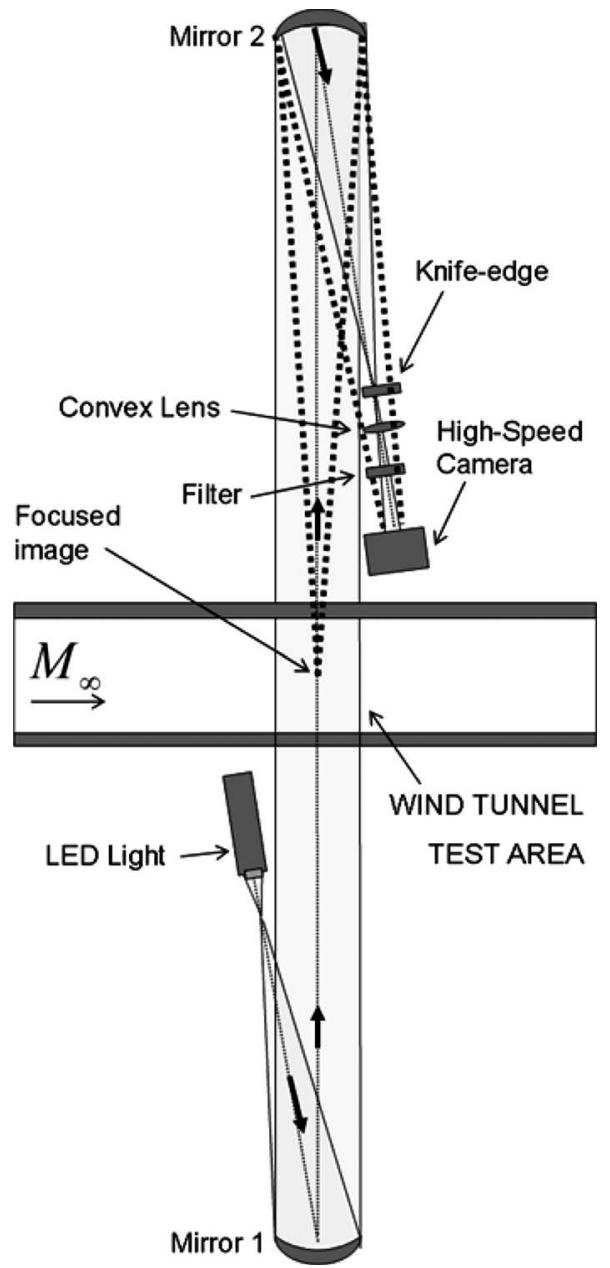

FIG. 1. Schematic diagram of high-speed schlieren optical arrangement. 


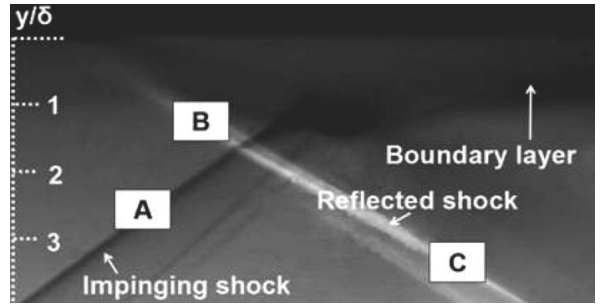

FIG. 2. Typical schlieren image of shock wave/turbulent boundary-layer interaction with separation indicating three different regions: A (incident shock), B (reflected shock upstream of impingement location), and C (reflected shock downstream of impingement location). Flow from left to right.

sonic Wind Tunnel at Mach 2.4 conditions. The high-speed camera has been operated at a frame rate of 10000 frames/s and with an image resolution of 512 pixels (width) $\times 256$ pixels (height), corresponding to 12 pixels $/ \mathrm{mm}$. The schlieren image has been captured directly onto the chip of the camera and polarized filters have been used to avoid image overexposure. Eight-bit images have been captured on a gray scale, with a time lapse of $100 \mu$ s between images. A horizontal knife edge has been used to obtain the first derivative of vertical density gradients.

A typical schlieren image is shown in Fig. 2, where the main features of the flow field are highlighted including the boundary layer and the reflected and impinging shocks. A reference distance $y / \delta$ is indicated, where $y$ is the vertical distance from the wall and $\delta$ is the boundary layer thickness.

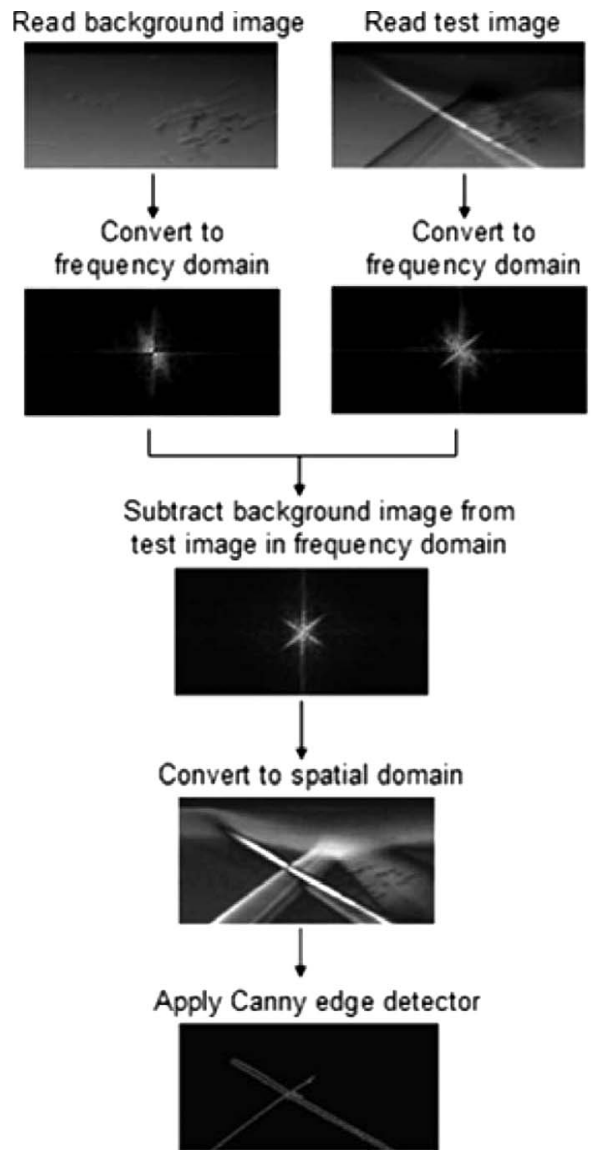

FIG. 3. Image processing routine including background image subtraction in the frequency domain to equally distinguish both dark and light shocks.

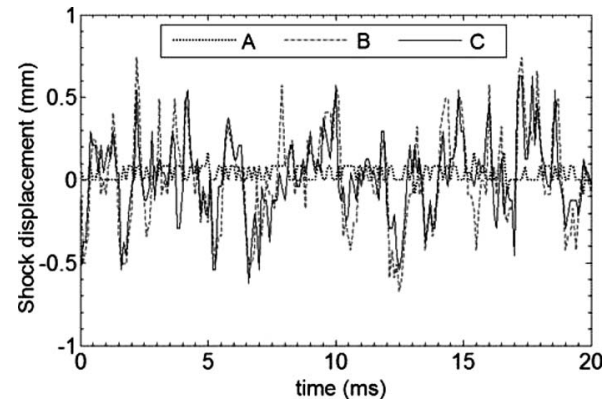

FIG. 4. Shock displacement ( $\mathrm{mm}$ ) as a function of time (ms), corresponding to locations A, B, and C from Fig. 2.

In order to quantify the shock wave positions, a number of image processing algorithms were developed to extract the required information from the images. The shock location is discernible as distinct dark or light regions in comparison with the background light intensity. Other flow features such as boundary layers or expansion regions are more gradual but also visible. ${ }^{10}$

A version of the Canny algorithm was applied to locate the shock positions. This algorithm is an edge detection method of common use in machine vision applications. ${ }^{11} \mathrm{~A}$ result of the algorithm's application is shown in Fig. 3. It was found to give enhanced performance in comparison with simpler edge detectors (Sobel, Prewitt, Roberts, and Laplace) when locating the shock position. This was mainly due to its reduced sensitivity to noise and its edge-thinning capability, which provided a clear shock location and avoided detection of unwanted edges. Shock position was determined by applying a suitable threshold to isolate the highest intensity gradients in the image, which once in binary form allowed the shocks to be distinguished as thin edges. ${ }^{12,13}$ From these edges the locations of the shocks were identified for each row in the image digital matrix $(256 \times 512)$. By
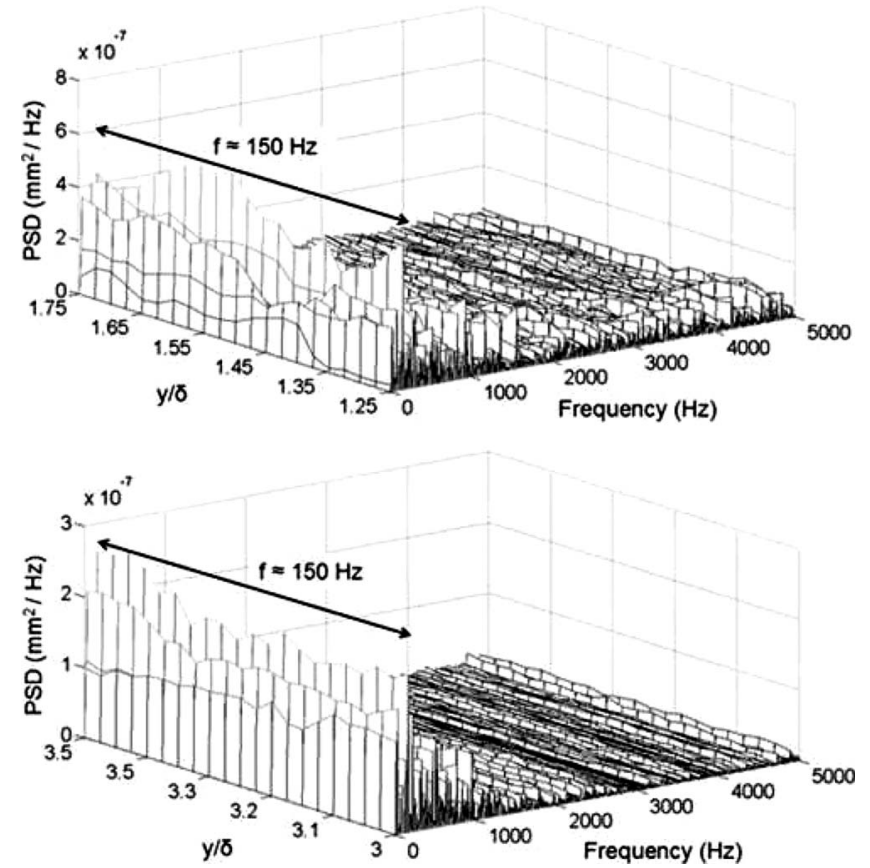

FIG. 5. Power spectral density plotted against frequency along locations (a) $y / \delta=1.25$ to $y / \delta=1.75$ and (b) $y / \delta=3.0$ to $y / \delta=3.5$ from Fig. 2 . 
processing a time series of images, the dominant frequencies in the shocks' motion were then obtained by means of spectral analysis using a Fourier transform based MATLAB toolbox. Shock unsteadiness was subsequently analyzed in different regions of the flow. In each case, sets of 1024 time-correlated schlieren images were taken at a frame rate of 10000 frames/s, giving an effective bandwidth of up to $5 \mathrm{kHz}$.

A typical time series of the location of the two shocks studied in the present application shows that the impinging shock is practically steady, whereas the position of the reflected shock changes with time (Fig. 4). The same plot also shows that the displacement of the reflected shock at locations upstream and downstream of the incident shock (B and $\mathrm{C}$, respectively, in Fig. 2) is in coherence most of the time. The corresponding spectral analysis of the reflected shock is shown in Fig. 5. With reference to these results and the image in Fig. 2, based on two independent sets of 1024 images and a frequency resolution of $9.8 \mathrm{~Hz}$, measurements have shown frequency peaks at approximately $150 \mathrm{~Hz}$ at locations $\mathrm{B}$ and $\mathrm{C}$. This is in agreement with previous wall pressure measurements at the location of the reflected shock's foot, which identified such unsteadiness to be in the order of $10^{2} \mathrm{~Hz}$ in similar studies. ${ }^{2,3}$
The method presented has therefore proven to be a reliable optical flow diagnostic for studies on shock wave unsteadiness. Its capability in obtaining flow measurements in regions not accessible by intrusive methods has also been shown.

${ }^{1}$ D. S. Dolling, Proceedings of the 31st AIAA Fluid Dynamics Conference and Exhibit, 2001 (unpublished), AIAA Paper No. 2001-2708.

${ }^{2}$ P. Dupont, C. Haddad, J. P. Ardissone, and J. F. Debieve, Aerosp. Sci. Technol. 9, 561 (2005).

${ }^{3}$ J.-P. Dussauge, P. Dupont, and J. F. Debieve, Aerosp. Sci. Technol. 10, 85 (2006).

${ }^{4}$ S. B. Verma, Exp. Fluids 32, 527 (2002).

${ }^{5}$ H. W. Liepmann and A. Roshko, Elements of Gasdynamics (Wiley, New York, 1957).

${ }^{6}$ B. Bretthauer, G. E. A. Meier, and B. Stasicki, Rev. Sci. Instrum. 62, 364 (1991).

${ }^{7}$ R. Haley and P. R. Smy, J. Phys. E 21, 1172 (1988).

${ }^{8}$ F. K. Lu and X. J. Liu, Opt. Eng. 36, 1935 (1997).

${ }^{9}$ A. Ben-Yakar and R. K. Hanson, Exp. Fluids 32, 652 (2002).

${ }^{10}$ G. S. Settles, Schlieren and Shadowgraph Techniques: Visualizing Phenomena in Transparent Media (Springer-Verlag, Berlin, 2001).

${ }^{11}$ R. C. Gonzalez, R. E. Woods, and S. L. Eddins, Digital Image Processing using MATLAB (Prentice Hall, New Jersey, 2004).

${ }^{12}$ J. Canny, IEEE Trans. Pattern Anal. Mach. Intell. 8, 679 (1986).

${ }^{13}$ L. Ding and A. Goshtasby, Pattern Recogn. 34, 721 (2001). 